\title{
Gastric Acid Suppression and Outcomes in Clostridium difficile Infection: A Population-Based Study
}

\author{
Sahil Khanna, MBBS; Scott L. Aronson, MD; Patricia P. Kammer, CCRP; \\ Larry M. Baddour, MD; and Darrell S. Pardi, MD, MS
}

\begin{abstract}
Objective: To evaluate the association of gastric acid suppression medications, including proton pump inhibitors and histamine type 2 blockers, with outcomes in patients with Clostridium difficile infection (CDI) in a population-based cohort.

Patients and Methods: To understand the association between acid suppression and outcomes in patients with CDI, we conducted a population-based study in Olmsted County, Minnesota, from January 1, 1991, through December 31, 2005. We compared demographic data and outcomes, including severe, severe-complicated, and recurrent CDI and treatment failure, in a cohort of patients with CDI who were treated with acid suppression medications with these outcomes in a cohort with CDI that was not exposed to acid-suppressing agents.

Results: Of 385 patients with CDI, 36.4\% were undergoing acid suppression (23.4\% with proton pump inhibitors, $13.5 \%$ with histamine type 2 blockers, and $0.5 \%$ with both). On univariate analysis, patients taking acid suppression medications were significantly older ( 69 vs 56 years; $P<.001$ ) and more likely to have severe ( $34.2 \%$ vs $23.6 \% ; P=.03$ ) or severecomplicated (4.4\% vs $2.6 \% \mathrm{CDI} ; P=.006$ ) infection than patients not undergoing acid suppression. On multivariable analyses, after adjustment for age and comorbid conditions, acid suppression medication use was not associated with severe or severecomplicated CDI. In addition, no association between acid suppression and treatment failure or CDI recurrence was found.

Conclusion: In this population-based study, after adjustment for age and comorbid conditions, patients with CDI who underwent acid suppression were not more likely to experience severe or severe-complicated CDI, treatment failure, or recurrent infection.
\end{abstract}

(C) 2012 Mayo Foundation for Medical Education and Research " Mayo Clin Proc. 2012;87(7):636-642

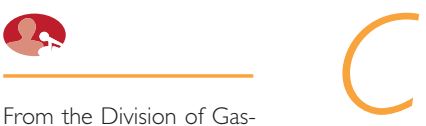

lostridium difficile infection (CDI) is the predominant cause of nosocomial infectious diarrhea. ${ }^{1,2}$ Striking and consistent increases troenterology and Hepatology (S.K., P.P.K., D.S.P.) and Division of Infectious Diseases (L.M.B.), Mayo Clinic, Rochester, MN; and Division of Gastroenterology, Hofstra North Shore-LIJ School of Medicine, New Hyde Park, NY (S.L.A.)

in incidence and severity of CDI have been documented. ${ }^{1-3} \mathrm{C}$ difficile infection ranges from mild to severe disease and can be complicated by recurrent illness, sepsis, need for critical care and/or surgical intervention, and death. Potentially novel risk factors associated with CDI have been identified, including those that circumvent the protective effect of stomach acid. These factors include the use of postpyloric enteral feeding or acid-suppressing medications, including proton pump inhibitors (PPIs) or histamine type $2\left(\mathrm{H}_{2}\right)$ receptor blockers. ${ }^{4,5}$ These data are supported by evidence that the vegetative form of $C$ difficile is sensitive to stomach acid. ${ }^{6}$ However, evidence on whether stomach acid kills $C$ difficile spores is conflicting. ${ }^{7}$

Regardless, several studies have identified exposure to PPIs as an independent risk factor associated with the development of CDI, and a systematic review of the topic concluded that gastric acid suppression therapy increases the risk of acquiring CDI 2-fold. ${ }^{8-10}$ However, these findings are bal- anced by reports that have not found an association between acid suppression medication use and risk of CDI. ${ }^{11,12}$ Furthermore, other studies have found that, after controlling for important confounders such as age, comorbid conditions, prior history of CDI, antibiotic exposure, and hospitalization, use of PPI or $\mathrm{H}_{2}$ blockers was not associated with the risk of developing CDI. ${ }^{13,14}$

With respect to outcomes, 3 retrospective studies have found an increased risk of recurrent CDI in patients taking a PPI, ${ }^{15-17}$ although a subgroup analysis of a large randomized controlled trial did not find a difference in the rates of recurrent CDI in patients with or without exposure to PPI and $\mathrm{H}_{2}$ receptor antagonists. ${ }^{18}$ We performed a populationbased study to examine outcomes in patients with CDI who were or were not receiving acid suppression medications at the time of CDI.

\section{PATIENTS AND METHODS}

\section{Study Population}

All cases of CDI occurring in residents of Olmsted County, Minnesota, from January 1, 1991, through 
December 31, 2005, were identified using the resources of the Rochester Epidemiology Project (REP). ${ }^{19,20}$ The REP diagnostic index was searched for the International Classification of Diseases, Ninth Revision (ICD-9) code for CDI (008.45) along with CDI as a microbiologic or clinical diagnosis (for both inpatients and outpatients). Medical records from virtually all sources of care available to Olmsted County residents are linked and accessible through the REP and contain all information about medical diagnoses, hospital admissions, surgical procedures, results of laboratory tests, results of imaging tests, and drug prescriptions. The REP enables investigators to follow up patients through their outpatient and hospitalization contacts across all local medical facilities, regardless of where the care was delivered and of insurance status. A central diagnostic index maintains records from all outpatient visits, emergency department visits, hospitalizations, nursing home stays, autopsy examinations, and death certificates for all residents since 1908.

All records of patients who had provided permission for their medical records to be used in research were reviewed. The REP database was searched for a listed CDI diagnosis and ICD-9 diagnosis code for CDI (008.45). Clinical notes, laboratory results, and endoscopy and histopathology reports were reviewed to confirm diagnoses. Records were reviewed to identify separate cases for individual patients and determine the acquisition setting (community vs hospital). The Mayo Clinic and Olmsted Medical Center institutional review boards approved the study.

\section{Case Definitions}

On the basis of recent recommendations, ${ }^{21,22}$ "definite" CDI was defined as 3 or more loose stools per 24 hours with a positive $C$ difficile stool toxin assay result or the presence of pseudomembranous colitis on endoscopy or histology reports. Infection was defined as hospital acquired if symptoms began more than 48 hours after admission or less than 4 weeks after discharge from a health care facility. Infection was defined as community acquired if symptom onset occurred in the community or within 48 hours of admission to a hospital, provided symptom onset was more than 12 weeks after the last discharge from a hospital. Indeterminate infection was defined as symptom onset between 4 and 12 weeks after last hospital dismissal, and 20 of the 385 cases (5.2\%) were included as community acquired in this study. Antibiotic exposure was defined as the use of oral or parenteral antibiotics in the 90 days preceding CDI diagnosis. Acid-suppressing medication use was defined as the concomitant use of either a PPI or an $\mathrm{H}_{2}$ receptor blocker at the time of CDI diagnosis.
According to the Infectious Diseases Society of America/Society for Healthcare Epidemiology of America guidelines, severe disease was defined by a peripheral white blood cell count of $15 \times 10^{9} / \mathrm{L}$ or a serum creatinine increase of $50 \%$ or greater from baseline. $^{22}$ This definition of severe CDI was based on expert opinion because it has not been prospectively validated in outcomes investigations; however, recent evidence supports this opinion that an elevated white blood cell count is associated with severe outcomes in CDI. ${ }^{23}$ Infection was classified as severe-complicated if it was associated with hypotension, sepsis, ileus, toxic megacolon, perforation, need for intensive care unit admission, surgery for a CDI-related complication (eg, megacolon, perforation, or refractory colitis), or death. ${ }^{22}$ Treatment failure was defined by persistent symptoms leading to a change in initial treatment within 14 days because of nonresponse or intolerable adverse effects. Recurrent CDI was defined as the diagnostic criteria of CDI being met within 8 weeks of a previous diagnosis after documented symptom resolution. ${ }^{24-26}$ Only the first CDI episode or those more than 8 weeks after the last episode were included in the analysis; recurrent CDI episodes were not included.

Comorbid conditions for all patients were assessed by calculating the crude and age-adjusted Charlson Comorbidity Index. ${ }^{27}$ The Charlson Comorbidity Index is composed of 19 comorbid conditions in 4 categories, and each category has a weighted score based on the adjusted risk of 1- and 10-year mortality. ${ }^{27} \mathrm{~A}$ higher score reflects a more severe comorbidity burden and an increased likelihood of mortality. A modified Charlson Comorbidity Index was also calculated after removing peptic ulcer disease to prevent confounding while using the index in multivariable regression models.

\section{Statistical Analyses}

Statistical analysis was performed using JMP statistical software, version 9.0.1 (SAS Institute Inc, Cary, NC). Descriptive analyses were performed for demographic characteristics and other clinical variables. The univariate associations of demographic and clinical characteristics with acid suppression medication use were assessed via contingency tables analyses ( $\chi^{2}$ test). Initial multivariable regression analysis was performed to evaluate the association of CDI outcomes (severe disease, severe-complicated disease, treatment failure, and recurrence) with acid suppression use while adjusting for demographic characteristics (age and sex) and clinically relevant covariates (Charlson Comorbidity Index and prior antibiotic use). Subsequent multivariable logistic regression models were performed to evaluate the as- 


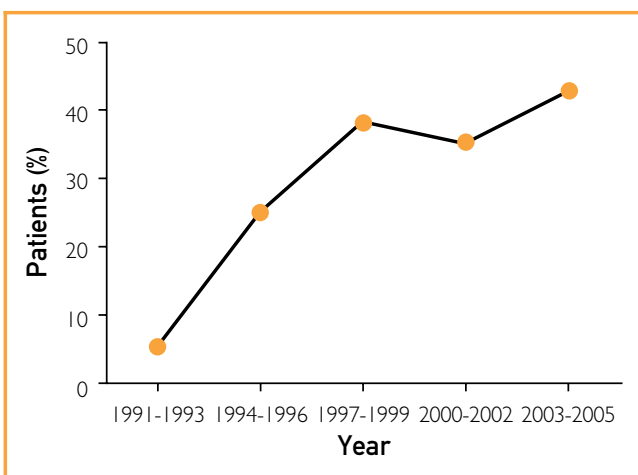

FIGURE. Time trends depicting a significant increase in the proportion of acid suppression medication use in patients with Clostridium difficile infection during the study period $(P=.001)$.

sociation of these outcomes with acid suppression medication use along with age and Charlson Comorbidity Index. Odds ratios (ORs) and 95\% confidence intervals (CIs) were calculated for both univariate and multivariable analyses.

\section{RESULTS}

\section{Patient Characteristics}

A total of 416 possible cases of CDI were identified; 31 (7.5\%) were excluded-30 because of a lack of a confirmatory stool assay and 1 because of a lack of authorization to access records for research. There were 385 definite CDI cases included in the study. The epidemiology of CDI in this cohort of patients has been previously described. ${ }^{28}$ The median age was 67.6 years (range, 10 days to 102 years), and 253 (65.7\%) were female. Most patients (204/385, $53.0 \%)$ were 65 years or older, and a substantial proportion of them (157/385, 40.8\%) had community-acquired infection. The overall age- and sexadjusted incidence of CDI was 25.2 per 100,000 person-years. ${ }^{28}$ Acid suppression medications were used by 140 (36.4\%) of them: 88 (22.9\%) taking PPIs alone, 50 (13.0\%) taking $\mathrm{H}_{2}$ blockers alone, and $2(0.5 \%)$ taking both agents. The proportion of patients with CDI taking acid suppression medications increased significantly during the study period from 5.3\% in 1991-1993 to $42.6 \%$ in $2003-2005$ $(P=.001$, Figure). Of the 140 patients undergoing acid suppression, 12 (8.6\%) did not have prior antibiotic exposure, and of the 245 patients not undergoing acid suppression, 38 (15.5\%) did not have prior antibiotic exposure $(P=.05)$.

Key demographic and clinical data in the 2 groups are compared in Table 1. Patients receiving acid suppression medications were significantly older, had a higher mean total and modified Charlson Comorbidity Index, and were more likely to have hospital-acquired infection than patients not taking these medications. Both groups had more females than males, and most had prior antibiotic exposure. Metronidazole was the most common initial treatment in both groups.

\begin{tabular}{|c|c|c|c|}
\hline Characteristic & $\begin{array}{l}\text { Received acid } \\
\text { suppression medications } \\
\qquad(n=140)\end{array}$ & $\begin{array}{l}\text { Did not receive acid } \\
\text { suppression medications } \\
\qquad(n=245)\end{array}$ & $P$ value \\
\hline Median age (y) (range) & $74(1-99 y)$ & $61(10 d-102 y)$ & $<.001$ \\
\hline Women & $87(62.1)$ & $166(67.8)$ & .26 \\
\hline Charlson Comorbidity Index, mean \pm SD & $3.43 \pm 2.89$ & $2.03 \pm 2.61$ & $<.001$ \\
\hline $\begin{array}{l}\text { Modified Charlson Comorbidity Index, } \\
\text { mean } \pm \text { SD }\end{array}$ & $3.22 \pm 2.78$ & $1.96 \pm 2.58$ & $<.001$ \\
\hline \multicolumn{4}{|l|}{ Patients by mode of acquisition } \\
\hline Hospital acquired & $90(64.3)$ & $102(4 \mid .6)$ & $<.001$ \\
\hline Nursing home acquired & $15(10.7)$ & $21(8.6)$ & .47 \\
\hline Community acquired & $35(25.0)$ & $122(49.8)$ & $<.001$ \\
\hline Patients exposed to antibiotics & $128(91.4)$ & $207(84.5)$ & .06 \\
\hline $\begin{array}{l}\text { Patients with metronidazole as their initial } \\
\text { treatment }\end{array}$ & $125(89.3)$ & $216(88.2)$ & .66 \\
\hline
\end{tabular}




\section{Outcomes of CDI}

On univariate analyses, patients taking acid suppression medications were more likely to have severe and severe-complicated infection than those not taking these medications. No differences were found in treatment failure or recurrence between these groups (Table 2).

When acid suppression medications were analyzed separately (PPI vs $\mathrm{H}_{2}$ blocker vs no acid suppression), patients taking these acid suppression medications were more likely to have severe-complicated CDI ( $10.2 \%$ vs $14.0 \%$ vs $4.1 \% ; P=.02)$ and trended toward being more likely to have severe CDI $(P=.09)$ compared with patients not taking acid suppression medications. No differences were seen in treatment failure or CDI recurrence. No differences were found in these outcomes in a head-to-head comparison of patients taking PPIs vs $\mathrm{H}_{2}$ blockers.

In initial multivariable logistic regression analysis (Table 3), including age in decades, sex, prior antibiotic exposure, crude Charlson Comorbidity Index, and acid suppression use as covariates, a higher Charlson Comorbidity Index was associated with the likelihood of having severe CDI, whereas age, sex, prior antibiotic exposure, and the use of acid suppression medications were not. Increasing age and Charlson Comorbidity Index were predictors of severe-complicated CDI, but acid suppression medication use was not. Increasing age was a predictor of treatment failure, but prior antibiotic exposure and acid suppression medication were not. None of the factors assessed were associated with the risk of recurrent infection.

In separate multivariable logistic regression models, when only age and acid suppression medication use were included, acid suppression was associated with severe-complicated CDI (adjusted OR, 2.34; 95\% CI, 1.03-5.56) but not severe CDI (adjusted OR, 1.48; 95\% CI, 0.92-2.39). However, when including Charlson Comorbidity Index in the model, the association between acid suppression and the likelihood of having severe-complicated
CDI was no longer seen (adjusted OR, 2.08; 95\% CI, 0.9-5.02). Similar results were obtained using a modified Charlson Comorbidity Index that removed peptic ulcer disease as a component of the score.

\section{DISCUSSION}

In this population-based study, which included both hospital- and community-acquired cases of $\mathrm{CDI}$, the use of acid suppression medications was associated with a risk of developing severe and severe-complicated infection on univariate analysis. However, after controlling for comorbid conditions, the use of these medications was no longer associated with the risk of severe or severe-complicated infection. Acid suppression medication use also was not associated with treatment failure or risk of recurrent infection. To our knowledge, this is the first population-based study assessing CDI outcomes associated with the concomitant use of acid suppression medications.

Medications that suppress gastric acid have been associated with alteration of gastrointestinal flora and increased susceptibility to gastrointestinal infections. ${ }^{29}$ Acid suppression medications are implicated in alteration of bowel flora of the upper and lower gastrointestinal tract. Bacteria in the small and large intestines increase in number in the conditions with decreased gastric acid. ${ }^{30,31}$ These medications are commonly prescribed, and there is emerging literature questioning the safety of PPIs. ${ }^{32,33}$ Many studies have addressed the association between the risk of $\mathrm{CDI}$ and use of acid suppression medications and have found that acid suppression is a risk factor associated with the development of CDI. ${ }^{4,34-37}$ However, it has not been conclusively proven that acid suppression with PPI is an independent risk factor associated with the development of CDI, and potential confounders could explain the observed associations. ${ }^{38-40}$ The vegetative form of $C$ difficile is susceptible to gastric acid, although spores are believed to be resistant. ${ }^{41} \mathrm{~A}$ potential mechanism to

\section{TABLE 2. Univariate Analysis of CDI Outcomes ${ }^{\mathrm{a}}$}

\begin{tabular}{|c|c|c|c|c|}
\hline Outcome & $\begin{array}{l}\text { No. (\%) of patients } \\
\text { undergoing acid suppression } \\
(n=\mid 40)\end{array}$ & $\begin{array}{l}\text { No. (\%) of patients not } \\
\text { undergoing acid suppression } \\
\qquad(n=245)\end{array}$ & $\begin{array}{l}\text { OR }(95 \% \mathrm{Cl}) \\
\text { (reference: no acid } \\
\text { suppression) }\end{array}$ & $P$ value \\
\hline Severe CDI & $48(34.3)$ & $58(23.7)$ & $1.68(1.06-2.65)$ & .02 \\
\hline Severe-complicated CDI & $17(12.1)$ & $10(4.1)$ & $3.24(\mid .44-7.31)$ & .003 \\
\hline Treatment failure ${ }^{b}$ & $26 / 134(19.4)$ & $49 / 238(20.6)$ & $0.93(0.54-1.58)$ & .78 \\
\hline Recurrent CDI & $37(26.4)$ & $79(32.2)$ & $0.75(0.47-1.19)$ & .22 \\
\hline
\end{tabular}




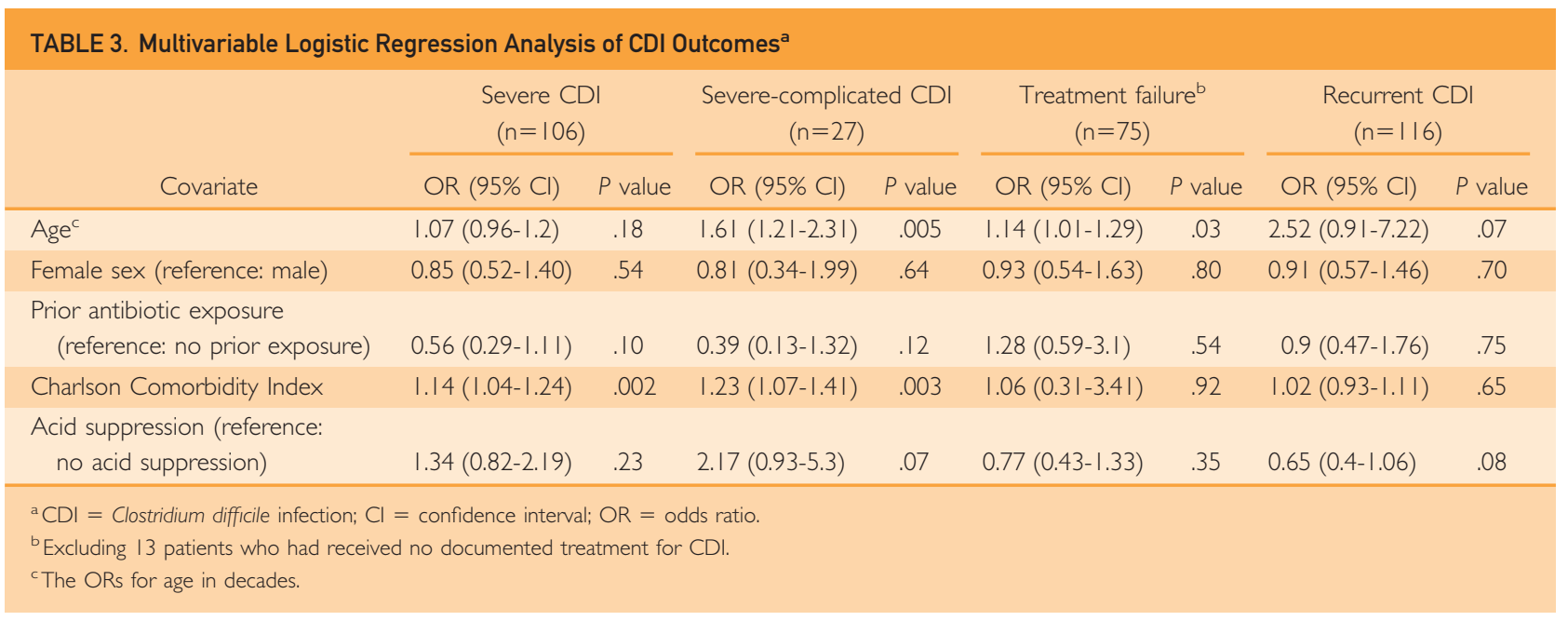

explain the association of acid suppression medications with the development of CDI is increased survival of the vegetative forms of $C$ difficile in response to decreased acid levels. ${ }^{41}$

There is a paucity of studies assessing the effect of concomitant acid suppression medication use on outcomes in patients with CDI. One hospital-based retrospective study revealed that $69 \%$ of CDI patients were taking PPIs, and of them, 21\% did not respond to CDI treatment and $41 \%$ had CDI recurrence. ${ }^{15}$ In this study, the risk of recurrence was 4-fold higher than in patients not taking acid suppression medication, but the difference in treatment failure was not significant. However, this study did not control for important confounders, including age and comorbid conditions, which may also be associated with the risk of recurrence. In a similar retrospective, hospital-based study, patients who received oral PPIs within 14 days of CDI diagnosis had a $42 \%$ increased risk of CDI recurrence. ${ }^{16}$ Recurrence in this study was defined by a positive stool test result, but symptoms were not accounted for, which could lead to falsely elevated recurrence rates because persistence of $C$ difficile toxin in the stool after treatment does not equate with recurrence. In our study, we were able to capture patient symptoms and define recurrent CDI as recurrent diarrhea after symptom resolution and the presence of a positive stool test result.

In contrast to these studies, a subgroup analysis of a recently published, large randomized controlled trial did not find a difference in the rates of recurrent CDI in patients with or without exposure to PPIs and $\mathrm{H}_{2}$ receptor antagonists. ${ }^{18}$ The association between acid suppression medications and the risk of severe and severe-complicated CDI was not evaluated in these studies.
Increased comorbidity burden and age are wellknown factors that adversely affect patient outcomes in general. ${ }^{27}$ In our results, univariate analyses suggested that concomitant acid suppression medication use was associated with the development of severe and severe-complicated CDI ( Table 2). However, after adjusting for age, Charlson Comorbidity Index, and other covariates, the association between severe and severe-complicated CDI and acid suppression was no longer present. Of interest, when only age was adjusted for, acid suppression was still associated with severe-complicated infection. However, when Charlson Comorbidity Index was further adjusted for, the association between acid suppression and severecomplicated infection was no longer statistically significant.

Our findings that increasing age and comorbidity burden are associated with worse outcomes in patients with CDI mirror previous studies. ${ }^{17,26,42}$ These patient factors should be incorporated in clinical decision making, perhaps influencing the choice of initial antibiotic therapy for CDI and also suggesting that these patients be monitored closely for complications from CDI. In addition, these confounding variables should be controlled for in any subsequent studies that assess the association between acid suppression and CDI incidence or outcomes.

The major strength of our study is that data have been collected during a 15-year period in a stable population. The resources of the REP permitted the identification of all cases of CDI in county residents and access to all the medical record information for each case. Because it was population based, our cohort was less prone to referral bias than previous hospital-based studies and included those with community-acquired CDI. 
Potential limitations of the study include its retrospective nature and the inability to assess indication for, duration of, and adherence with acid suppression medication use. Thus, we are unable to analyze the association between duration of acid suppression medication and CDI outcomes. Another limitation was the lack of $C$ difficile strain information, which was not available from our laboratory during the study.

\section{CONCLUSION}

This population-based based study found that patients with CDI who were taking acid suppression medications were more likely to develop severe and severe-complicated infection by univariate analysis. However, by adjusting for key confounders, including age and comorbid conditions, these associations were no longer significant. Patients with CDI who have a good indication for acid suppression medications may continue to take these agents. Additional studies are needed to confirm our observation that the use of these medications is not associated with complications of CDI.

Abbreviations and Acronyms: $\quad C D I=$ Clostridium difficile infection; $\mathrm{H}_{2}$ = histamine type 2; ICD-9 = International Classification of Diseases, Ninth Revision; PPI = proton pump inhibitor; REP $=$ Rochester Epidemiology Project

Grant Support: This research was supported in part by an unrestricted research grant from ViroPharma and by the Rochester Epidemiology Project (grant number ROIAG034676 from the National Institute of Aging; Principal Investigator: Walter A. Rocca, MD).

Correspondence: Address to Darrell S. Pardi, MD, MS, Division of Gastroenterology and Hepatology, Mayo Clinic, 200 First St SW, Rochester, MN 55905 (pardi.darrell@ mayo.edu).

\section{REFERENCES}

1. Khanna S, Pardi DS. The growing incidence and severity of Clostridium difficile infection in inpatient and outpatient settings. Expert Rev Gastroenterol Hepatol. 20 10;4(4):409-4I6.

2. Kelly CP, LaMont JT. Clostridium difficile-more difficult than ever. N Engl J Med. 2008;359( | 8): 1932-1940.

3. Zilberberg MD. Clostridium difficile-related hospitalizations among US adults, 2006. Emerg Infect Dis. 2009; I 5(I): I22-124.

4. Al-Tureihi F, Hassoun A, Wolf-Klein G, Isenberg H. Albumin, length of stay, and proton pump inhibitors: key factors in Clostridium difficile-associated disease in nursing home patients. J Am Med Dir Assoc. 2005;6(2): 105- 108.

5. Dubberke E, Reske K, Yan Y, Olsen M, McDonald L, Fraser V. Clostridium difficile-associated disease in a setting of endemicity: identification of novel risk factors. Clin Infect Dis. 2007; 45( I 2): 1543-1549.

6. Rao A, Jump RLP, Pultz NJ, Pultz MJ, Donskey CJ. In vitro killing of nosocomial pathogens by acid and acidified nitrite. Antimicrob Agents Chemother. 2006;50(1 1):3901-3904.
7. Wilson $\mathrm{KH}$, Sheagren JN, Freter R. Population dynamics of ingested Clostridium difficile in the gastrointestinal tract of the Syrian hamster. J Infect Dis. 1985; I5I (2):355-36।.

8. Dial S, Delaney JAC, Barkun AN, Suissa S. Use of gastric acid-suppressive agents and the risk of community-acquired Clostridium difficile-associated disease. JAMA. 2005;294(23): 2989-2995.

9. Leonard J, Marshall JK, Moayyedi P. Systematic review of the risk of enteric infection in patients taking acid suppression. Am J Gastroenterol. 2007; I02(9):2047-2056; quiz 2057.

10. Aseeri M, Schroeder T, Kramer J, Zackula R. Gastric acid suppression by proton pump inhibitors as a risk factor for Clostridium difficile-associated diarrhea in hospitalized patients. Am J Gastroenterol. 2008; 103(9):2308-2313.

11. Metz DC. Clostridium difficile colitis: wash your hands before stopping the proton pump inhibitor. Am J Gastroenterol. 2008; 103(9):2314-2316.

12. Shah S, Lewis A, Leopold D, Dunstan F, Woodhouse K Gastric acid suppression does not promote clostridial diarrhoea in the elderly. QJM. 2000;93(3): I75-I81.

13. Kyne L, Sougioultzis S, McFarland LV, Kelly CP. Underlying disease severity as a major risk factor for nosocomial Clostridium difficile diarrhea. Infect Control Hosp Epidemiol. 2002; 23(I I):653-659.

14. Pepin J, Saheb N, Coulombe MA, et al. Emergence of fluoroquinolones as the predominant risk factor for Clostridium difficile-associated diarrhea: a cohort study during an epidemic in Quebec. Clin Infect Dis. 2005;4I (9): I 254- 1260.

15. Cadle R, Mansouri M, Logan N, Kudva D, Musher D. Association of proton-pump inhibitors with outcomes in Clostridium difficile colitis. Am J Health Syst Pharm. 2007;64(22):2359-2363.

16. Linsky A, Gupta K, Lawler EV, Fonda JR, Hermos JA. Proton pump inhibitors and risk for recurrent Clostridium difficile infection. Arch Intern Med. 20 10; 170(9):772-778.

17. Kim JW, Lee KL, Jeong JB, et al. Proton pump inhibitors as a risk factor for recurrence of Clostridium-difficile-associated diarrhea. World J Gastroenterol. 20 I0; 16(28):3573-3577.

18. Linsky A, Gupta K, Hermos JA. Fidaxomicin for Clostridium difficile infection. N Engl J Med. 2011;364(19):1875; author reply 1875-1876

19. Melton LJ III. History of the Rochester Epidemiology Project. Mayo Clin Proc. 1996;7I (3):266-274.

20. St Sauver JL, Grossardt BR, Yawn BP, Melton LJ III, Rocca WA. Use of a medical records linkage system to enumerate a dynamic population over time: the Rochester epidemiology project. Am J Epidemiol. 20 I I; I73(9): I059- 1068.

21. McDonald LC, Coignard B, Dubberke E, Song X, Horan T, Kutty PK; Ad Hoc Clostridium difficile Surveillance Working Group. Recommendations for surveillance of Clostridium difficile-associated disease. Infect Control Hosp Epidemiol. 2007; 28(2): | 40- 145.

22. Cohen SH, Gerding DN, Johnson S, et al. Clinical practice guidelines for Clostridium difficile infection in adults: 2010 update by the Society for Healthcare Epidemiology of America (SHEA) and the Infectious Diseases Society of America (IDSA). Infect Control Hosp Epidemiol. 20 10;3। (5):431-455.

23. Lungulescu OA, Cao W, Gatskevich E, Thabano L, Stratidis JG. CSI: a severity index for Clostridium difficile infection at the time of admission. J Hosp Infect. 201 1;79(2); 15 I- 154.

24. Loo VG, Poirier L, Miller MA, et al. A predominantly clona multi-institutional outbreak of Clostridium difficile-associated diarrhea with high morbidity and mortality. N Engl J Med. 2005;353(23):2442-2449. 
25. McDonald LC, Coignard B, Dubberke E, et al. Recommendations for surveillance of Clostridium difficile-associated disease. Infect Control Hosp Epidemiol. 2007;28(2): | 40-145.

26. Pepin J, Alary ME, Valiquette L, et al. Increasing risk of relapse after treatment of Clostridium difficile colitis in Quebec, Canada. Clin Infect Dis. 2005;40( I I): 1591-1597.

27. Charlson ME, Pompei $P$, Ales KL, MacKenzie CR. A new method of classifying prognostic comorbidity in longitudinal studies: development and validation. J Chronic Dis. 1987;40(5): 373-383.

28. Khanna S, Pardi DS, Aronson SL, et al. The epidemiology of community-acquired Clostridium difficile infection: a population-based study. Am J Gastroenterol. 2012;107(1):89-95.

29. Williams C. Occurrence and significance of gastric colonization during acid-inhibitory therapy. Best Pract Res Clin Gastroenterol. 200 I; I5(3):5 I I-52।.

30. Kanno T, Matsuki T, Oka M, et al. Gastric acid reduction leads to an alteration in lower intestinal microflora. Biochem Biophys Res Commun. 2009;38I (4):666-670.

31. Bavishi C, Dupont HL. Systematic review: the use of proton pump inhibitors and increased susceptibility to enteric infec tion. Aliment Pharmacol Ther. 20 | 1;34(| |- |2):|269-1281.

32. Lodato F, Azzaroli F, Turco L, et al. Adverse effects of proton pump inhibitors. Best Pract Res Clin Gastroenterol. 20 10;24(2): |93-201.

33. Sataloff RT. Proton pump inhibitors: adverse effects. Ear Nose Throat J. 2010;89(12):574-576

34. Dial S, Delaney JAC, Schneider V, Suissa S. Proton pump inhibitor use and risk of community-acquired Clostridium difficile-associated disease defined by prescription for oral vancomycin therapy. CMAJ. 2006; 175(7):745-748.
35. Cunningham R. Proton pump inhibitors and the risk of Clostridium difficile-associated disease: further evidence from the community. CMAJ. 2006; I75(7):757.

36. Cunningham R, Dale B, Undy B, Gaunt N. Proton pump inhibitors as a risk factor for Clostridium difficile diarrhoea. Hosp Infect. 2003:54(3):243-245.

37. Dalton B, Lye-Maccannell T, Henderson E, Maccannell D, Louie T. Proton pump inhibitors increase significantly the risk of Clostridium difficile infection in a low-endemicity, non-outbreak hospital setting. Aliment Pharmacol Ther. 2009;29(6):626634.

38. Famularo G, Gasbarrone L, De Simone C. Proton pump inhibitors and the risk for Clostridium difficile infection. JAMA. 2009;302(1):31-32; author reply 32.

39. Beaulieu M, Williamson D, Pichette G, Lachaine J. Risk of Clostridium difficile-associated disease among patients receiving proton-pump inhibitors in a Quebec medical intensive care unit. Infect Control Hosp Epidemiol. 2007;28( I I): | 305- 307.

40. Lowe DO, Mamdani MM, Kopp A, Low DE, Juurlink DN Proton pump inhibitors and hospitalization for Clostridium difficile-associated disease: a population-based study. Clin Infect Dis. 2006;43(I0): I 272- 1276

41. Jump RL, Pultz MJ, Donskey CJ. Vegetative Clostridium difficile survives in room air on moist surfaces and in gastric contents with reduced acidity: a potential mechanism to explain the association between proton pump inhibitors and C. difficileassociated diarrhea? Antimicrob Agents Chemother. 2007;5 I (8): 2883-2887.

42. Garey KW, Sethi S, Yadav Y, DuPont HL. Meta-analysis to assess risk factors for recurrent Clostridium difficile infection. $J$ Hosp Infect. 2008;70(4):298-304. 